\title{
Affective and Motivational Responses to 3D Body Imaging
}

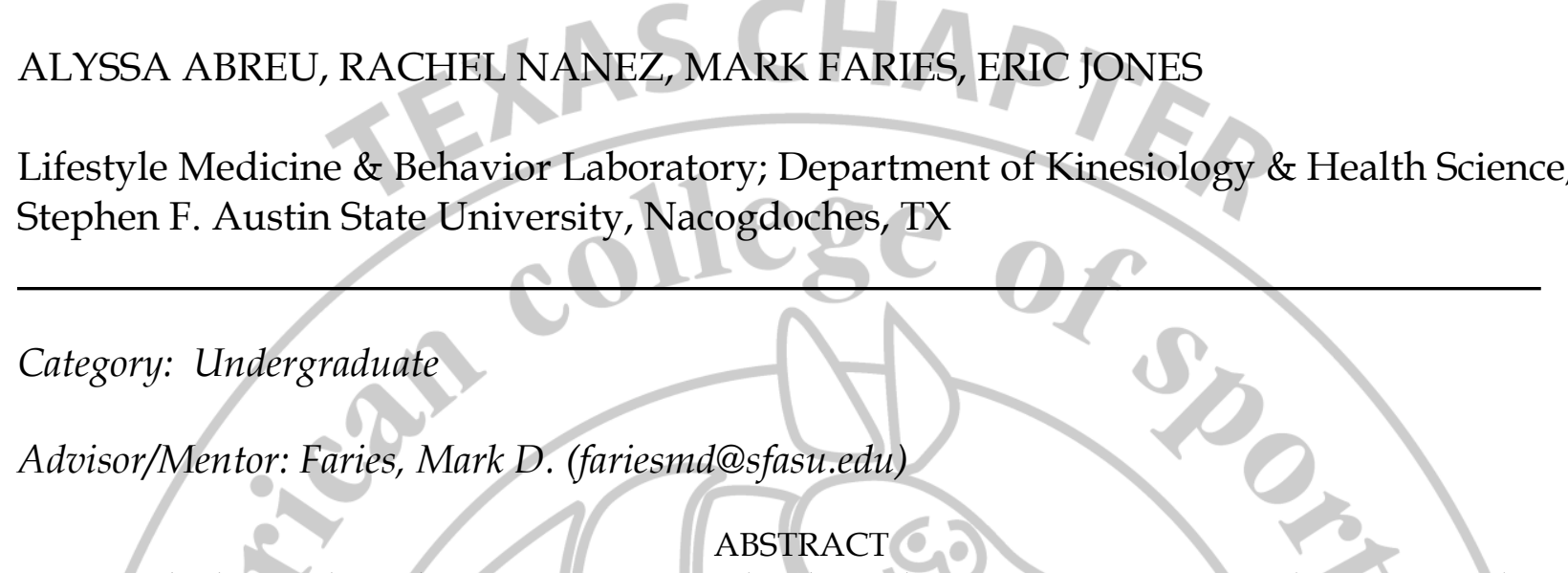

Common body weight and composition testing has been shown to impact emotional, motivational, and behavioral responses. Three-dimensional body imaging is a relatively new technology to be utilized in medical and fitness settings to provide detailed body image feedback to the patient or client, while encouraging motivation to control body weight, fat, and shape. However, such responses to 3D body imaging have not been examined. To examine the acute affective and motivational responses to 3D body imaging (Fit3D) in a sample of college-aged women $(N=32)$, classified as either normal weight (NW; $n=$ 13) or overweight/obese (OWOB; $n=19$ ). Positive and negative affect were assessed with the Positive (PA) and Negative Affect (NA) Schedule (PANAS; range: 1-5), and one item assessed motivation to lose weight (range: 1-10). These variables were assessed immediately before and after a single 3D body imaging session. Participants had 1-minute to examine their 3D body scan, and then continued to examine their scan during all post-measures. Overall, PA did not change pre- to post-scan $(3.11 \pm .98$ to $3.18 \pm 1.07$, $p>.05)$, nor did NA $(1.52 \pm .58$ to $1.63 \pm .89, p>.05)$. In addition, there was not a time by BMI (NW versus OWOB $)$ interaction for both PA and NA. Overall, motivation to lose weight slightly increased $(6.41 \pm 2.78$ to $7.09 \pm 2.75, p=.001$ ). There was not an interaction of time by BMI, with both groups increasing over time. However, there was a between-subjects effect with OWOB holding higher pre-motivation to lose weight than NW participants (7.63 versus 4.62 , respectively; $p<.01$ ). Within the present sample of collegeaged women, a 3D body image scanning session did not appear impact PA or NA responses, but did produce a slight increase in motivation to lose body weight in participants classified as NW or OWOB. This research provides foundational insight to future research and the use of this novel technology for health behavior change in fitness and clinical settings.

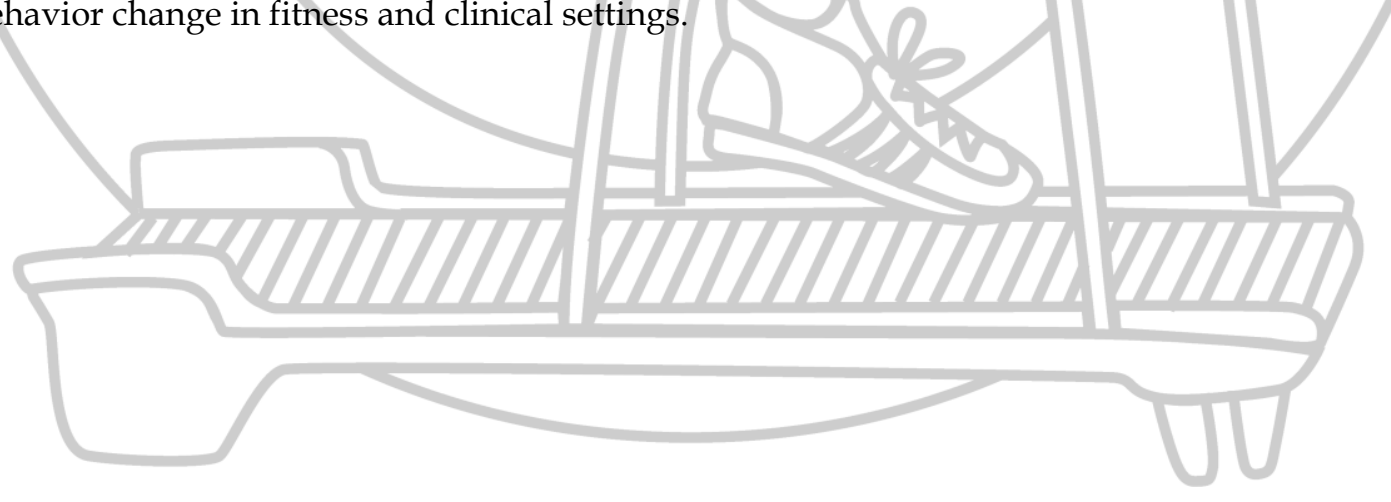

